\title{
Continuum of maternity care in Zambia: a national representative survey
}

\author{
Quraish Sserwanja $^{1^{*}}$ (D), Milton W. Musaba ${ }^{2} \mathbb{D}$, Linet M. Mutisya ${ }^{3}$ (D) Emmanuel Olal ${ }^{4}$ and David Mukunya ${ }^{5,6}$ (D)
}

\begin{abstract}
Background: Globally, over half of maternal deaths are related to pregnancy-related complications. Provision of a continuum of care during pregnancy, childbirth and the postnatal period results in reduced maternal and neonatal morbidity and mortality. Hence this study determined the prevalence of the continuum of care and its determinants among women in Zambia.

Methods: We used weighted data from the Zambian Demographic and Health Survey (ZDHS) of 2018 for 7325 women aged 15 to 49 years. Multistage stratified sampling was used to select study participants. Complete continuum of care was considered when a woman had; at least four antenatal care (ANC) contacts, utilized a health facility for childbirth and had at least one postnatal check-up within six weeks. We conducted multivariable logistic regression to explore continuum of care in Zambia. All our analyses were done using SPSS version 25.
\end{abstract}

Results: Of the 7,325 women, 38.0\% (2787/7325) (95\% confidence interval (CI): 36.9-39.1) had complete continuum of maternal healthcare. Women who had attained tertiary level of education (adjusted odds ratio (AOR): 1.93, 95\% Cl: 1.09-3.42) and whose partners had also attained tertiary level of education (AOR: 2.58, 95\% Cl: 1.54-4.32) were more likely to utilize the whole continuum of care compared to those who had no education. Women who initiated ANC after the first trimester (AOR: $0.46,95 \% \mathrm{Cl}: 0.39-0.53$ ) were less likely to utilize the whole continuum of care compared to those who initiated in the first semester. Women with exposure to radio (AOR: 1.58, 95\% Cl: 1.271.96) were more likely to utilize the whole continuum of care compared to those who were not exposed to radio. Women residing in the Western province were less likely to utilize the entire continuum of care compared to those in the other nine provinces.

Conclusion: Level of education of the women and of their partners, early timing of ANC initiation, residing in other provinces other than the Western province, and exposure to information through radio were positively associated with utilization of the entire continuum of care. Improving literacy levels and promoting maternity services through radio may improve the level of utilization of maternity services.

Keywords: Continuum of care, Antenatal care, Postnatal care, Childbirth, Utilization, Women and Zambia

\section{Background}

Globally, at least half of maternal mortality results from pregnancy complications, and over 277,300 (94\%) of these deaths occur in low- and middle-income countries and roughly two-thirds $(196,000)$ of the deaths take

\footnotetext{
* Correspondence: qura661@gmail.com; qsserwanja@sd.goal.ie

${ }^{1}$ Programs Department, GOAL, Arkaweet Block 65 House No. 227, Khartoum, Sudan

Full list of author information is available at the end of the article
}

place in sub-Saharan Africa [1, 2]. A woman's lifetime risk of maternal death in sub-Saharan Africa is 1 in 59, far higher than the risk in all low-income countries, estimated at 1 in 160 [3]. Majority of these preventable maternal and perinatal deaths occur in the intrapartum and immediate postpartum period [4] and have been linked to behavioral risk factors [5]. The direct maternal deaths and could be prevented by strengthening and improving access to the whole continuum of maternity care $[6,7]$.

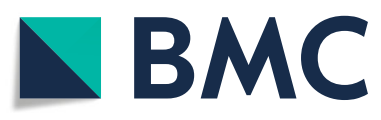

(c) The Author(s). 2021 Open Access This article is licensed under a Creative Commons Attribution 4.0 International License, which permits use, sharing, adaptation, distribution and reproduction in any medium or format, as long as you give appropriate credit to the original author(s) and the source, provide a link to the Creative Commons licence, and indicate if changes were made. The images or other third party material in this article are included in the article's Creative Commons licence, unless indicated otherwise in a credit line to the material. If material is not included in the article's Creative Commons licence and your intended use is not permitted by statutory regulation or exceeds the permitted use, you will need to obtain permission directly from the copyright holder. To view a copy of this licence, visit http://creativecommons.org/licenses/by/4.0/ The Creative Commons Public Domain Dedication waiver (http://creativecommons.org/publicdomain/zero/1.0/) applies to the data made available in this article, unless otherwise stated in a credit line to the data. 
Additionally, 1 in 12 children die before reaching the age of five years and most of these deaths occur in the neonatal period $[8,9]$. Neonatal mortality contributes to almost $45 \%$ of all under- 5 childhood deaths worldwide $[8,9]$. Globally, 2.4 million neonates died in 2019 which approximates to 6,700 neonatal deaths daily [10]. SubSaharan Africa has the highest neonatal mortality rate estimated at 27 deaths per 1,000 live births $[10,11]$.

Ensuring accelerated progress towards achieving sustainable development goal (SDG) 3 needs global health focus on programs and policies targeting reduction of preventable maternal, newborn and child mortality and morbidity [4]. Limited access and low utilization of maternal healthcare services are some of the main predictors of negative maternal health outcomes in low income countries such as Zambia [12,13]. It is estimated that if effective health measures are ensured during childbirth and in the first week of life, we could avert over $80 \%$ of maternal deaths and $66 \%$ of neonatal deaths globally $[4,14]$. To ensure this, cost effective measures within the reach of low and middle income countries such as continuum of care are required [4]. Continuum of care ensures uninterrupted flow in the care at all stages hence improving maternal and child health outcomes [15]. Complete exposure to the continuum of care compared to separate healthcare ensures that utilization at each stage builds on the effect of the previous stage of healthcare access.

Reducing maternal mortality requires a dramatic increase in utilization of health facilities during childbirth and quality of maternal health care delivered [16]. The World Health Organization (WHO) global guidelines recommend that women deliver at facilities with the capacity to manage emergencies [17]. Consistent with WHO guidelines, the Zambian guidelines recommend all women to have childbirth in a health facility [18]. In our study, we focused on the continuity of care over time from pregnancy through childbirth to the postpartum period. We looked at antenatal care (ANC) attendance, health facility utilization during childbirth and postnatal care (PNC).

Adequate, timely and quality ANC has been shown to improve birth preparedness and increase skilled birth attendance [6]. Further, it enables early identification and management of pregnancy related illnesses and complications $[6,19]$. Health facility utilization during childbirth increases the chances of a safe, clean environment that is wellequipped with skilled personnel, drugs, and supplies necessary for effective and timely management of childbirth complications [4]. Additionally, PNC enables timely identification of any complications that might affect maternal and neonatal health outcomes [4]. These three interventions are crucial in lowering maternal and neonatal mortality rates, thus the need to provide all of them in a continuum of care [20].
As per the National Health Strategic Plan for 20172021, Zambia set a target to decrease the maternal mortality ratio (MMR) from 398 to 162 maternal deaths per 100,000 live births by 2021 [21]. Over the last two decades, Zambia has achieved a steady reduction in the MMR which currently stands at 252 deaths per 100,000 live births [22]. However, this MMR is almost four times higher than the SDG 3 target of 70 deaths per 100,000 live births by 2030 [9]. Most studies have focused on exploring factors associated with use of individual maternal health services, but few have explored the utilization of care as a continuum. To ensure effective continuum of care program designing and implementation, it is crucial to understand the healthcare seeking gaps along the continuum pathway and the factors associated with the gaps. Our study aimed at describing the factors influencing the continuum of care during pregnancy, at childbirth and during postpartum period among women in Zambia. The findings will inform the design of programs and strategies aimed at improving level of utilization of the continuum of care, in order to reduce the maternal and neonatal mortality.

\section{Methods \\ Study design and participants}

The Zambian Demographic and Health Survey (ZDHS) was a nationally representative cross-sectional study conducted using validated questionnaires between $18^{\text {th }}$ July 2018 and $24^{\text {th }}$ January 2019 [22]. It is a periodic survey that is carried out every five years as part of the MEASURE DHS global survey and collects information on demographic, health, and nutrition indicators. The survey used stratified two-stage cluster sampling design that resulted in the random selection of a representative sample of 13,625 households [22]. The first stage involved 545 cluster (sample points) selection which consisted of enumeration areas using a sampling frame that was used during the 2010 census of population and housing $(\mathrm{CPH})$ [22]. Zambia is divided into 10 provinces which are further divided into districts, constituencies, and wards [22]. Wards, the smallest administrative units, were further divided during the $2010 \mathrm{CPH}$ into census supervisory areas which were later divided into enumeration areas. Enumeration areas were selected with a probability proportional to their size within each sampling stratum. Systematic sampling was applied to select households in the second stage. A detailed explanation of the sampling process is available in the ZDHS 2018 report [22].

Women aged 15-49 years who were either permanent residents or visitors who had stayed in the selected households the night before the survey were eligible for interviews and a total of 13,683 women being interviewed. Four questionnaires were used in the survey and 
our study used data that was obtained using validated women's and household questionnaires. Our secondary analysis included women of reproductive age (1549 years) who had given birth in the last five years preceding the survey that yielded a weighted sample of 7,325 women. Written informed consent was provided by all participants of the survey. Written permission to access the whole ZDHS database was obtained through DHS program website at this address https://dhsprogram.com/

\section{Variables}

\section{Outcome variables}

Complete continuum of maternal healthcare is the outcome variable, Complete continuum of care was constructed into binary variable with complete coded as 1 and incomplete coded as 0 . Complete continuum of maternal healthcare was considered when a woman reported having had:

1. Had at least four ANC (ANC4+) contacts during the most recent childbirth.

2. Utilized a health facility during the most recent childbirth and

3. Had at least one postnatal check-up within six weeks after childbirth $[4,23]$

Continuum of care was considered discontinued if the woman skipped any of the steps above [4, 24, 25]. Additionally, having four or more ANC contacts was considered as continued care during pregnancy while utilizing health facility during childbirth with four or more ANC contacts was considered as continued care during delivery.

\section{Independent variables}

Independent variables were categorized into women, partners' and household characteristics which were chosen basing on previous studies [26-28] and availability in the ZDHS database.

\section{Partner characteristics}

This included the partner's highest level of education (no education, primary, secondary, and tertiary).

\section{Household characteristics}

Wealth index of household (categorized into quintiles: richest, richer, middle poorer and poorest), type of residence (urban and rural), and province/region that included the official 10 provinces of Zambia. Wealth index is a measure of relative household economic status and was calculated by DHS from information on household asset ownership using principal component analysis [22, 29].

\section{Women's characteristics}

Age (15-24, 25-34, and 35-49), level of education (no education, primary, secondary, and tertiary), exposure to newspapers/magazines, radio and TV (yes and no), preceding birth interval ( less than 24 months and 24 months and above), parity (one, two and three or more), age at firth birth (less than 20 and 20 and above), ANC timing (first trimester and second or third trimester), marital status (married and not married), age at first sex (less than 18 and 18 and above), working status (working and not working) and decision making for seeking healthcare (involved and not involved).

\section{Statistical analysis}

We used the SPSS analytic software version 25.0 complex samples package. Weighted data was used to account for the unequal probability sampling in different strata. Frequency distributions were used to describe the background characteristics of the women. Bivariable and multivariable logistic regression analyses were conducted to identify the association among various independent variables at all the three levels of continuum of care. Independent variables found significant at p-value less than 0.25 in the bivariable analysis were included in the multivariable models. We presented two models; the primary model, which adjusted for only factors without missing data, and the secondary model, which included all the factors (with and without missing data) and adjusted for those with missing data. The secondary model included residence, provinces, exposure to mass media, working and marital status, level of education, wealth index, timing of ANC initiation, preceding birth interval, age at first sexual intercourse and first birth and healthcare seeking decision making. Adjusted odds ratios (AOR), 95\% confidence intervals (CI) and p-values were calculated with statistical significance level set at p-value $<0.05$. Sensitivity analysis was done by analyzing continued care during pregnancy with having eight or more ANC contacts as the outcome.

\section{Results}

The mean age of women was 28.5 (standard deviation (SD) 7.5$)$ years with majority (41.2\%) of them being 25 34 years. while that of the mothers was 28.80 (SD 6.83). Majority of the women resided in rural areas (61.6\%), were married (75.2\%), had primary education (49.1\%), initiated ANC after first trimester (62.0\%), had their first sexual intercourse when they were less than 18 years (74.3\%) and had their first pregnancy when they were below 20 years (68.1\%). Of the 7,325 women, $38.0 \%$ (2787/7325) (95\% CI: 36.9-39.1) had complete continuum of maternity care (four or more ANC contacts, health facility utilization during childbirth and postnatal care attendance). Further analysis showed 64.1\% (4651/ 
7262) (95\% CI: 63.1-65.2) had four or more ANC contacts (continued care during pregnancy) while 57.3\% (4199/7325) (95\% CI: 56.2-58.5) had continued care at delivery (four or more ANC contacts and utilized a health facility for childbirth). More detailed characteristics of study participants are shown in Table 1.

\section{Factors associated with complete continuum of maternity} care

Results from multivariable logistic regression (Table 2) showed that province, timing of ANC initiation, exposure to radio, partners' and women's level of education were associated with complete continuum of maternity.

Details about factors associated with four or more ANC4 contacts (continued care during pregnancy) and four or more ANC contacts and health facility utilization at childbirth (continued care at childbirth) are shown in supplementary files 1 and 2 .

\section{Sensitivity analysis \\ Continued care during pregnancy (Eight or more ANC contacts)}

Only 91 (1.2\%, 95\% CI: 1.0-1.5) women had attended at least eight ANC contacts. In the multivariable analysis, only three factors were associated with eight or more ANC contacts which included: age, residence, and initiation of ANC timing. Younger women aged 15-24 years were 243\% (AOR: 3.43 95\% CI: 1.46-8.05) more likely to have eight or more ANC contacts compared to their older counterparts aged 35-49 years. Women in urban areas were $245 \%$ more likely (AOR: 3.45 , $95 \%$ CI: $1.48-$ 8.04) to have eight or more ANC contacts compared to women in rural areas. Women who initiated ANC contacts after the first trimester were $92 \%$ less likely (AOR: 0.08, 95\% CI: 0.03-0.18) to have eight or more ANC contacts compared to women who initiated ANC in the first trimester.

\section{Discussion}

Of the 7,325 women, $38.0 \%$ had continuum of maternity care. The level of continued care reduced at every stage of care from $64.1 \%$ during pregnancy to $57.3 \%$ at child birth and $38 \%$ complete continuum of care during postpartum period. The largest dropout occurred between delivery and the postnatal period. This finding of complete continuum of care is lower than findings from Cambodia and Egypt studies [20, 23] and higher than findings from studies conducted in Pakistan, Tanzania, Ethiopia and Ghana [4, 24, 30, 31].

We found that province, timing of ANC initiation, exposure to radio, and the level of education of women and their partners were associated with complete continuum of maternity care. Although preceding birth interval was not associated with complete continuum of care, it was found to be significantly associated with continued care during pregnancy and at childbirth.

Women who initiated ANC after the first trimester were less likely to have complete continuum of care compared to those who initiated ANC in the first trimester. This was observed at all stages of accessing care (continued care during pregnancy and at childbirth). Initiating ANC early enables women to have more ANC contacts thus ability to complete the first continuum which is the first step in ensuring complete continuum of care [32, 33]. The increased likelihood of more ANC contacts associated with early ANC initiation leads to adequate health education sessions regarding the benefits of skilled birth attendance, postnatal care and creates rapport between the health workers and the women [32-34]. This further increases the likelihood of the utilization of the subsequent components of maternity continuum of care. ANC timing has been shown to be associated with complete continuum of care in previous studies [24, 32, 35].

Province was a significant factor at every level of care. However, the association became stronger at the complete continuum of care level. Women belonging to the Western province were less likely to have complete continuum of care compared to the women in the other nine provinces. Variations in the complete continuum of care utilization was large with Lusaka province accounting for the highest percent of women at $17.5 \%$ with complete continuum of care, Copperbelt second at $15.1 \%$, Eastern third at $14.4 \%$ and Western province with the lowest percentage at $3.9 \%$. This finding is probably related to the availability and ease of access to quality reproductive, maternal and neonatal health services. Yan et al. showed that urban areas of Lusaka and Copperbelt provided maternity services of a higher quality compared to the other regions [16]. Furthermore, projects such as the Saving Mothers, Giving Life (SMGL) initiative implemented in provinces of Eastern and Luapula aimed at addressing the second delay to accessing health care by pregnant women. This increased the proportion of women utilizing health facilities for childbirth between 2012 and 2016 [17]. Since our study included women who had childbirth in the preceding five years of the survey, the impact SMGL programme could in part explain the observed higher likelihood of continuum of care in Luapula and Eastern provinces. Western province women had the least likelihood of complete continuum of care. Western province is characterized by difficult geographical and climatic conditions which has greatly affected agricultural production hence affecting household income [36]. Secondly, over $80 \%$ of the population is considered to be poor of which $70 \%$ of these are women [36]. These poverty levels can affect access to care hence delayed ANC initiation, access to good 
Table 1 Background characteristics of women who had given birth in the last 5 years

\begin{tabular}{lll}
\hline Characteristics & $\mathbf{N = 7 3 2 5}$ & $\%$ \\
\hline Parity & & \\
Three or more & 3884 & 53.0 \\
Two & 1440 & 19.7 \\
One & 2001 & 27.3 \\
Residence & & 38.4 \\
$\quad$ Urban & 2811 & 61.6 \\
Rural & 4513 &
\end{tabular}

\section{Provinces}

\section{Central}

Copper belt

Eastern

Luapula

640

Lusaka

1219

Muchinga

433

Northern

North Western

Southern

Western

Working status

$$
\begin{aligned}
& \text { Not working } \\
& \text { Working }
\end{aligned}
$$

Marital status

Not Married

Married

5506

Education Level

No Education

689

Primary Education

Secondary Education

Tertiary

Wealth Index

Poorest

Poorer

Middle

Richer

Richest

\section{5}

3490

1819

3595

2726

316

1676

1527

1390

1471

1262

\section{Age in years}

15-24

25-34

3020

35-49

1699

ANC timing ${ }^{a}$

Second or third trimester

First trimester

2690

$\%$

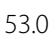

19.7

27.3

38.4

61.6

\section{7}

13.2

13.4

8.7

16.6

5.9

8.4

5.5

12.9

6.5

6.5

52.4

47.6

24.8

24.8

75.2

9.4

9.4

49.1

37.2

4.3

22.9

20.8

19.0

20.1

$$
17.2
$$

Exposure to Newspapers
Table 1 Background characteristics of women who had given birth in the last 5 years (Continued)

\begin{tabular}{lll}
\hline Characteristics & $\mathbf{N}=\mathbf{7 3 2 5}$ & $\%$ \\
\hline Yes & 1217 & 16.6 \\
No & 6108 & 83.4 \\
Exposure to Radio & & \\
Yes & 3278 & 44.8 \\
No & 4047 & 55.2
\end{tabular}

Exposure to TV

$\begin{array}{lll}\text { Yes } & 2581 & 35.2\end{array}$

$\begin{array}{lll}\text { No } & 4744 & 64.8\end{array}$

Preceding Birth Interval ${ }^{\mathrm{b}}$

24 months and above $\quad 4666 \quad 63.7$

Less than 24 months $\quad 822 \quad 11.2$

Partner's education ${ }^{c}$

$\begin{array}{lll}\text { No education } & 322 & 4.4\end{array}$

Primary $2011 \quad 27.5$

Secondary $\quad 2530 \quad 34.5$

$\begin{array}{lll}\text { Tertiary } & 444 & 6.1\end{array}$

Age at first sex

Less than 18 years $\quad 5444 \quad 74.3$

18 years and above $\quad 1880 \quad 25.7$

Healthcare seeking decision ${ }^{d}$

Involved $\quad 4374 \quad 59.7$

$\begin{array}{lll}\text { Not involved } & 1132 & 15.5\end{array}$

\section{Age at first birth}

Less than 20 years $\quad 4987 \quad 68.1$

20 years and above $\quad 2338 \quad 31.9$

ANC attendance ${ }^{\mathrm{e}}$

$\begin{array}{lll}\text { Less than } 4 & 2611 & 35.9 \\ 4 \text { and above } & 4651 & 64.1\end{array}$

Place of delivery

Home $\quad 951 \quad 13.0$

$\begin{array}{lll}\text { Health Facility } & 6373 & 87.0\end{array}$

Postnatal care

$\begin{array}{lll}\text { No } & 2691 & 36.7\end{array}$

Yes $4633 \quad 63.2$

${ }^{\mathrm{a}}$ Missing 93, ${ }^{\mathrm{b}}$ missing 1836, ${ }^{\mathrm{C}}$ missing 2018, ${ }^{\mathrm{d}}$ missing 1819, ${ }^{\mathrm{e}}$ missing 63

quality education and affordability of mass media channels such as radios which factors have been shown to be associated with exposure to the complete continuum of care. This association between the region of residence and continuum of care has be reported in previous studies conducted in Ghana and Cambodia [20,25].

Exposure to radio was positively associated with continued care during pregnancy and complete continued of care. Women who were exposed to radio were more 
Table 2 Predictors of complete continuum of maternity care in Zambia

\begin{tabular}{|c|c|c|c|c|c|c|}
\hline Characteristics & Complete continued care $n=2787$ & P-value & $\begin{array}{l}\text { Crude model } \\
\text { COR }(95 \% \mathrm{Cl})\end{array}$ & P-value & $\begin{array}{l}\text { Primary Model } \\
\text { AOR }(95 \% \mathrm{Cl})\end{array}$ & $\begin{array}{l}\text { Secondary Model } \\
\text { AOR }(95 \% \mathrm{Cl})\end{array}$ \\
\hline Parity & & 0.223 & & 0.408 & & \\
\hline Three or more & $1445(51.9)$ & & 1 & & & \\
\hline Two & $551(19.7)$ & & $1.04(0.89-1.23)$ & & & \\
\hline One & $791(28.4)$ & & $1.10(0.96-1.27)$ & & & \\
\hline Residence & & 0.003 & & 0.082 & & \\
\hline Rural & $1656(59.4)$ & & 1 & & 1 & 1 \\
\hline Urban & $1130(40.6)$ & & $1.16(0.98-1.37)$ & & $0.99(0.79-1.26)$ & $1.05(0.79-1.40)$ \\
\hline Provinces & & $<0.001$ & & $<0.001$ & & \\
\hline Western & $109(3.9)$ & & 1 & & 1 & 1 \\
\hline Copperbelt & $422(15.1)$ & & $2.61(1.81-3.75)$ & & $2.30(1.58-3.35)$ & $3.11(1.85-5.23)$ \\
\hline Eastern & $400(14.4)$ & & $2.32(1.62-3.34)$ & & $2.22(1.55-3.19)$ & $2.91(1.79-4.74)$ \\
\hline Luapula & $267(9.6)$ & & $2.41(1.59-3.67)$ & & $2.22(1.49-3.29)$ & $2.90(1.74-4.82)$ \\
\hline Lusaka & $488(17.5)$ & & $2.25(1.56-3.26)$ & & $1.95(1.33-2.87)$ & $2.81(1.67-4.75)$ \\
\hline Muchinga & $209(7.5)$ & & $3.16(2.09-4.76)$ & & $3.19(2.17-4.69)$ & $4.08(2.46-6.75)$ \\
\hline Northern & $272(9.8)$ & & $2.69(1.61-4.49)$ & & $2.66(1.60-4.41)$ & $3.63(1.92-6.85)$ \\
\hline North Western & $164(5.9)$ & & $2.31(1.62-3.31)$ & & $2.40(1.69-3.40)$ & $3.88(2.29-6.57)$ \\
\hline Southern & $264(9.5)$ & & $1.31(0.87-1.97)$ & & $1.25(0.84-1.87)$ & $1.48(0.91-2.43)$ \\
\hline Central & $191(6.9)$ & & $1.44(0.98-2.12)$ & & $1.32(0.90-1.95)$ & $1.86(1.15-3.03)$ \\
\hline Working status & & 0.041 & & 0.128 & & \\
\hline Not working & $1416(50.8)$ & & 1 & & 1 & 1 \\
\hline Working & $1370(49.2)$ & & $1.10(0.97-1.26)$ & & $1.05(0.93-1.19)$ & $0.89(0.74-1.06)$ \\
\hline Marital status & & 0.006 & & 0.022 & & \\
\hline Not Married & $643(23.1)$ & & 1 & & 1 & - \\
\hline Married & $2143(76.9)$ & & $1.17(1.02-1.33)$ & & $1.10(0.96-1.26)$ & - \\
\hline Education Level & & $<0.001$ & & $<0.001$ & & \\
\hline No Education & $198(7.1)$ & & 1 & & & 1 \\
\hline Primary Education & $1316(47.2)$ & & $1.43(1.14-1.79)$ & & & $1.29(1.01-1.64)$ \\
\hline Secondary Education & $1091(39.1)$ & & $1.65(1.33-2.05)$ & & & $1.21(0.91-1.61)$ \\
\hline Tertiary & $182(6.5)$ & & $3.34(2.32-4.82)$ & & & $1.93(1.09-3.42)$ \\
\hline Wealth Index & & $<0.001$ & & 0.052 & & \\
\hline Poorest & $611(21.9)$ & & 1 & & 1 & 1 \\
\hline Poorer & $550(19.7)$ & & $0.98(0.83-1.16)$ & & $0.93(0.78-1.11)$ & $0.88(0.71-1.10)$ \\
\hline Middle & $515(18.5)$ & & $1.03(0.84-1.25)$ & & $0.97(0.79-1.18)$ & $0.91(0.70-1.18)$ \\
\hline Richer & 547 (19.6) & & $1.03(0.81-1.31)$ & & $0.88(0.65-1.19)$ & $0.94(0.62-1.43)$ \\
\hline Richest & $563(20.2)$ & & $1.40(1.09-1.81)$ & & $0.92(0.66-1.30)$ & $0.79(0.48-1.29)$ \\
\hline Age in years & & 0.721 & & 0.777 & & \\
\hline $35-49$ & $644(23.1)$ & & 1 & & & \\
\hline $25-34$ & 1165 (41.8) & & $1.03(0.88-1.20)$ & & & \\
\hline $15-24$ & $978(35.1)$ & & $0.98(0.85-1.14)$ & & & \\
\hline ANC timing & & $<0.001$ & & $<0.001$ & & \\
\hline First trimester & $1353(48.6)$ & & 1 & & & 1 \\
\hline Second or third trimester & $1429(51.4)$ & & $0.45(0.40-0.51)$ & & & $0.46(0.39-0.53)$ \\
\hline Newspaper Exposure & & $<0.001$ & & 0.001 & & \\
\hline
\end{tabular}


Table 2 Predictors of complete continuum of maternity care in Zambia (Continued)

\begin{tabular}{|c|c|c|c|c|c|c|}
\hline Characteristics & Complete continued care $n=2787$ & P-value & $\begin{array}{l}\text { Crude model } \\
\text { COR }(95 \% \mathrm{Cl})\end{array}$ & P-value & $\begin{array}{l}\text { Primary Model } \\
\text { AOR }(95 \% \mathrm{CI})\end{array}$ & $\begin{array}{l}\text { Secondary Model } \\
\text { AOR }(95 \% \mathrm{CI})\end{array}$ \\
\hline No & $2237(80.3)$ & & 1 & & 1 & 1 \\
\hline Yes & $550(19.7)$ & & $1.43(1.15-1.77)$ & & $1.09(0.88-1.35)$ & $1.10(0.86-1.41)$ \\
\hline Exposure to Radio & & $<0.001$ & & $<0.001$ & & \\
\hline No & $1347(48.3)$ & & 1 & & 1 & 1 \\
\hline Yes & $1440(51.7)$ & & $1.57(1.38-1.79)$ & & $1.43(1.23-1.68)$ & $1.58(1.27-1.96)$ \\
\hline Exposure to TV & & $<0.001$ & & 0.002 & & \\
\hline No & $1705(61.2)$ & & 1 & & 1 & 1 \\
\hline Yes & $1082(38.8)$ & & $1.29(1.10-1.51)$ & & $0.96(0.77-1.18)$ & $0.92(0.73-1.16)$ \\
\hline Preceding Birth Interval & & 0.001 & & 0.006 & & \\
\hline Less than 24 months & $263(12.9)$ & & 1 & & & 1 \\
\hline 24 months and above & $1778(87.1)$ & & $1.31(1.08-1.59)$ & & & $1.23(0.99-1.52)$ \\
\hline Partner's education & & $<0.001$ & & $<0.001$ & & \\
\hline No education & $93(4.5)$ & & 1 & & & 1 \\
\hline Primary & $719(34.5)$ & & $1.37(0.95-1.96)$ & & & $1.31(0.88-1.94)$ \\
\hline Secondary & $1012(48.6)$ & & $1.64(1.17-2.29)$ & & & $1.40(0.96-2.05)$ \\
\hline Tertiary & $258(12.4)$ & & $3.42(2.32-5.03)$ & & & $2.58(1.54-4.32)$ \\
\hline Age at first sex & & $<0.001$ & & 0.001 & & \\
\hline 18 years and above & $794(28.5)$ & & 1 & & 1 & 1 \\
\hline Less than 18 years & $1993(71.5)$ & & $0.79(0.69-0.91)$ & & $1.01(0.85-1.19)$ & $1.15(0.93-1.43)$ \\
\hline Healthcare seeking decision & & $<0.001$ & & 0.006 & & \\
\hline Not involved & $385(18.0)$ & & 1 & & & 1 \\
\hline Involved & $1758(82.0)$ & & $1.30(1.08-1.58)$ & & & $1.24(0.99-1.55)$ \\
\hline Age at first birth & & $<0.001$ & & 0.001 & & \\
\hline 20 years and above & $978(35.1)$ & & 1 & & 1 & 1 \\
\hline Less than 20 years & $1808(64.9)$ & & $0.79(0.69-0.90)$ & & $0.92(0.78-1.07)$ & $0.99(0.80-1.23)$ \\
\hline
\end{tabular}

bold= Significant at p-value $<0.05$, Secondary model adjusted for residence, provinces, exposure to mass media, working and marital status, level of education, wealth index, timing of ANC initiation, preceding birth interval, age at first sexual intercourse and first birth and healthcare seeking decision making

likely to have more ANC contacts and complete continuum of care. The influence of media on access to maternal healthcare services could be attributed to the role media houses play in reducing knowledge gaps by sensitizing women on the benefits of healthcare seeking and utilization which leads to positive attitudes, challenges negative social norms and improves health seeking behavior [37, 38]. As a result, women exposed to mass media have been shown to be more likely to initiate ANC early in pregnancy and even have more contacts in our analysis and in previous studies $[38,39]$. This positive behavior possibly increases their likelihood to have adequate information from the health education sessions which promotes early recognition of danger signs and the need to seek for care from a skilled provider at the earliest opportunity [40]. Furthermore, people who are exposed to media are more likely to be educated, have discussions with others which interpersonal interactions contribute greatly in challenging negative norms that might affect health seeking and hence lead to positive health seeking behavioral change [40, 41]. Exposure to media has been shown in previous studies to have a positive association with continuum of care $[4,39]$.

Women and those with partners who had attained at least a tertiary level of education were more likely to have complete continuum of care compared to their uneducated counterparts. Sialubanje et al. showed that husbands influenced decisions on the utilisation of health services by pregnant women in Zambia [5]. This is mainly attributed to the centrality of husbands' roles within the Zambian culture, as family heads and often sole bread winners [5]. Sialubanje et al. also found that availability of funds for birth preparation, presence of a relative as home caretakers and people to work in the field when the woman is away, determined husbands' decision on whether their wives were to have childbirth from health facilities [5]. Higher levels of education have been shown to be associated with better maternal health 
literacy, more receptivity to new health related information, increased awareness of available health resources, better decision-making abilities and more financial resources increase their likelihood of accessing maternal healthcare services [32, 42]. With improved maternal health literacy, women and their partners become more informed about maternal health care issues which enables them to make positive health care decisions [43]. In most parts of sub-Saharan Africa including Zambia, the patriarchal system is practiced, hence men have a crucial role in the decision-making process, household resource control and allocation which affects women's access to healthcare [25]. Education has been shown to help challenge negative norms and lead to better healthcare decision making hence educated men are more likely to support their women to have continuum of care [25]. Therefore, the government of Zambia needs to intensify education to post- secondary level and also improve or start maternal health programs targeting the less educated women and their partners. Education has been shown in previous studies to be associated with continuum of care $[4,25,32]$.

\section{Strengths and limitations}

This is the foremost nationwide analysis that explores continuum of maternity care services. Therefore, it can be used as a yardstick and motivation for further studies on related subject matter in order to ensure effective maternal healthcare program implementation. Secondly, we used the most current nationally representative data hence the findings are generalizable to women in Zambia. However, use of cross-sectional data only enables associations to be established but not causal relationships. Sixty-three $(0.9 \%)$ of the 7325 women could not recall the number of ANC contacts they had and these were categorized among those who discontinued care which might have slightly reduced the percentage of women who had complete continuum of care. Lastly, the possibility of recall bias due to self-reported answers and lack of data on factors such as: distance of residence from health center, availability of emergency obstetric and newborn care services, presence of Safe Motherhood Action Groups, and the role of maternity waiting homes.

\section{Conclusion}

This study demonstrated only two out of five women were able to utilize the entire continuum of maternity care services. Level of education of the women and that of their partners (tertiary), early timing of ANC initiation, residing in other provinces other than the Western province, and exposure to radio were positively associated with completion of maternity continuum of care. Thus, we recommend the different stakeholders engaged in maternal health care to focus on women whose levels of education and that of their partners are below tertiary especially those residing in the Western province to ensure utilization of the entire continuum of maternity care. Utilization of mass media especially radio to sensitize the population on the benefits of continuum of care should be encouraged with great attention given for early initiation of ANC, increasing the number of ANC contacts, health facility and skilled birth attendance during childbirth and postnatal care.

\section{Abbreviations \\ AOR: Adjusted Odds Ratio; Cl: Confidence Interval; COR: Crude Odds Ratio; DHS: Demographic Health Survey; ZDHS: Zambia Demographic Health Survey; OR : Odds Ratio; SD: Standard Deviation; WHO : World Health Organization; SPSS: Statistical Package for Social Science; ANC: Antenatal care; PNC: Post-natal care}

\section{Supplementary Information}

The online version contains supplementary material available at https://doi. org/10.1186/s12884-021-04080-1

Additional file 1.

Additional file 2

Acknowledgements

We thank the MEASURE DHS program for availing us with the data and Dr. Daniel Mwamba of the Centre for Infectious Disease Research Zambia for reviewing the manuscript.

Authors' contributions

QS Conceived the idea, drafted the manuscript, performed analysis and interpreted the results. MWM, LMM, EO and DM participated in the design of the study and helped in results interpretation and writing. All authors read and approved the final manuscript.

Funding

No funding was obtained for this study.

Availability of data and materials

The data set used is publicly available upon permission from MEASURE DHS website (URL: https://www.dhsprogram.com/data/available-datasets.cfm).

\section{Declarations}

Ethics approval and consent to participate

High international ethical standards are ensured for MEASURE DHS surveys as ethical approval from the country is obtained from a national ethical review board and local authorities before implementing the survey and wellinformed verbal consent is sought from the respondents prior to data collection $[29,44]$. For the 2018 ZDHS, ethical approval was obtained from the Inner City Fund (ICF) and the Zambia Tropical Diseases Research Centre (TDRC), institutional review boards (IRBs) [45]. All methods of data collection were performed in accordance with the relevant guidelines and regulations. This data set was obtained from the MEASURE DHS website (URL: https:// www.dhsprogram.com/data/available-datasets.cfm) after getting their permission and no formal ethical clearance was obtained since we conducted secondary analysis of publicly available data.

Consent for publication

Not applicable.

Competing interests

All authors declare that they have no competing interests. 


\section{Author details}

'Programs Department, GOAL, Arkaweet Block 65 House No. 227, Khartoum, Sudan. ${ }^{2}$ Department of Obstetrics and Gynaecology, Busitema University, Mbale, Uganda. ${ }^{3}$ Maternal and Child Health Project, Swedish Organization for Global Health, Mayuge, Uganda. ${ }^{4}$ Yotkom Medical Centre, Kitgum, Uganda. ${ }^{5}$ Department of Community and Public Health, Busitema University, Mbale, Uganda. ${ }^{6}$ Sanyu Africa Research Institute, Mbale, Uganda.

Received: 19 February 2021 Accepted: 29 August 2021

Published online: 05 September 2021

\section{References}

1. Alkema L, Chou D, Hogan D, Zhang S, Moller A-B, Gemmill A, et al. Global, regional, and national levels and trends in maternal mortality between 1990 and 2015, with scenario-based projections to 2030: a systematic analysis by the UN Maternal Mortality Estimation Inter-Agency Group. Lancet. 2016; 387(10017):462-74

2. WHO. Maternal mortality. https://www.who.int/news-room/fact-sheets/deta il/maternal-mortality. Accessed 30 Dec 2020

3. World Health Organization (WHO). Trends in Maternal Mortality: 1990 to 2013. Estimates by WHO, UNICEF, UNFPA, The World Bank and the United Nations Population Division. 2014.

4. Iqbal S, Maqsood S, Zakar R, Zakar MZ, Fischer F. Continuum of care in maternal, newborn and child health in Pakistan: analysis of trends and determinants from 2006 to 2012. BMC Health Serv Res. 2017;17(1):189. https://doi.org/10.1186/s12913-017-2111-9.

5. Sialubanje C, Massar K, van der Pijl MSG, Kirch EM, Hamer DH, Ruiter RAC. Improving access to skilled facility-based delivery services: Women's belief on facilitators and barriers to the utilisation of maternity waiting homes in rural Zambia. Reprod Health. 2015;12:61.

6. Ekholuenetale M, Nzoputam Cl, Barrow A, Onikan A. Women's enlightenment and early antenatal care initiation are determining factors for the use of eight or more antenatal visits in Benin: further analysis of the Demographic and Health Survey. J Egypt Public Health Assoc. 2020;95(1):13.

7. Sserwanja Q, Musaba MW, Mukunya D. Prevalence and factors associated with modern contraceptives utilization among female adolescents in Uganda. BMC Womens Health. 2021:21(1):61.

8. Golding N, Burstein R, Longbottom J, Browne AJ, Fullman N, OsgoodZimmerman A, et al. Mapping under-5 and neonatal mortality in Africa, 2000-15: a baseline analysis for the Sustainable Development Goals. Lancet. 2017:390(10108):2171-82.

9. $\mathrm{WHO}$ |SDG 3: Ensure healthy lives and promote wellbeing for all at all ages. WHO. http://www.who.int/sdg/targets/en/. Accessed 15 Dec 2020

10. UNICEF. Neonatal mortality. https://data.unicef.org/topic/child-survival/ neonatal-mortality/. Accessed 15 Jan 2021.

11. Baqui AH, Mitra DK, Begum N, Hurt L, Soremekun S, Edmond K, et al. Neonatal mortality within 24 hours of birth in six low- and lower-middleincome countries. Bull World Health Organ. 2016;94(10):752-758B.

12. Sialubanje C, Massar K, Hamer DH, Ruiter RAC. Personal and environmental predictors of the intention to use maternal healthcare services in Kalomo, Zambia. Health Educ Res. 2014;29(6):1028-40.

13. Sialubanje C, Massar K, Horstkotte L, Hamer DH, Ruiter RAC. Increasing utilisation of skilled facility-based maternal healthcare services in rural Zambia: the role of safe motherhood action groups. Reprod Health. 2017; 14(1):81.

14. World Health Organization. Newborns: reducing mortality - Fact sheet, January 2016. Geneva: World Health Organization; 2016.

15. Kerber KJ, de Graft-Johnson JE, Bhutta ZA, Okong P, Starrs A, Lawn JE. Continuum of care for maternal, newborn, and child health: from slogan to service delivery. Lancet (London, England). 2007;370(9595):1358-69.

16. Yan LD, Mwale J, Straitz S, Biemba G, Bhutta Z, Ross JF, et al. Equity dimensions of the availability and quality of reproductive, maternal and neonatal health services in Zambia. Trop Med Int Health. 2018;23(4):433-45.

17. Ngoma T, Asiimwe AR, Mukasa J, Binzen S, Serbanescu F, Henry EG, et al. Addressing the Second Delay in Saving Mothers, Giving Life Districts in Uganda and Zambia: Reaching Appropriate Maternal Care in a Timely Manner. Glob Health Sci Pract. 2019;7(Suppl 1):S68-84.

18. Scott NA, Henry EG, Kaiser JL, Mataka K, Rockers PC, Fong RM, et al. Factors affecting home delivery among women living in remote areas of rural Zambia: a cross-sectional, mixed-methods analysis. Int J Women's Health. 2018;10:589-601.
19. Sserwanja Q, Kawuki J. Prevalence of Underweight and Associated Factors among Lactating Women in Ethiopia: A Mini-review. J Adv Med Med Res. 2020;32(8):1-9 https://doi.org/10.9734/jammr/2020/v32i830459.

20. Wang W, Hong R. Levels and determinants of continuum of care for maternal and newborn health in Cambodia-evidence from a populationbased survey. BMC Pregnancy Childbirth. 2015;15:62.

21. Kaiser $\mathrm{L}$, McGlasson KL, Rockers PC, Fong RM, Ngoma T, Hamer DH, et al. Out-of-pocket expenditure for home and facility-based delivery among rural women in Zambia: a mixed-methods, cross-sectional study. Int J Women's Health. 2019;11:411-30.

22. Zambia Statistics Agency - ZSA, Ministry of Health - MOH, University Teaching Hospital Virology Laboratory - UTH-VL, ICF: Zambia Demographic and Health Survey 2018. In. Lusaka, Zambia: ZSA, MOH, UTH-VL and ICF; 2020.

23. Hamed AF, Roshdy E, Sabry M. Egyptian status of continuum of care for maternal, newborn, and childhealth: Sohag Governorate as an example. Int J Med Med Sci. 2018;7:6

24. Mohan D, LeFevre AE, George A, Mpembeni R, Bazant E, Rusibamayila N, et al. Analysis of dropout across the continuum of maternal health care in Tanzania: findings from a cross-sectional household survey. Health Policy Plan. 2017;32(6):791-9.

25. Yeji F, Shibanuma A, Oduro A, Debpuur C, Kikuchi K, Owusu-Agei S, et al. Continuum of Care in a Maternal, Newborn and Child Health Program in Ghana: Low Completion Rate and Multiple Obstacle Factors. PLoS One. 2015;10(12):e0142849.

26. Muthuri SK, Francis CE, Wachira LJ, Leblanc AG, Sampson M, Onywera VO, et al. Evidence of an overweight/obesity transition among school-aged children and youth in Sub-Saharan Africa: a systematic review. PLoS One. 2014;9(3):e92846.

27. Tchoubi S, Sobngwi-Tambekou J, Noubiap JJ, Asangbeh SL, Nkoum BA, Sobngwi E. Prevalence and Risk Factors of Overweight and Obesity among Children Aged 6-59 Months in Cameroon: A Multistage, Stratified Cluster Sampling Nationwide Survey. PLoS One. 2015:10(12):e0143215.

28. Danquah Fl, Ansu-Mensah M, Bawontuo V, Yeboah M, Udoh RH, Tahiru M, et al. Risk factors and morbidities associated with childhood obesity in subSaharan Africa: a systematic scoping review. BMC Nutr. 2020;6(1):37.

29. Rutstein SO, Staveteig S. Making the Demographic and Health Surveys Wealth Index comparable. In: DHS Methodological Reports No 9. Rockville, Maryland, USA: ICF International; 2014.

30. Chaka EE, Parsaeian M, Majdzadeh R. Factors Associated with the Completion of the Continuum of Care for Maternal, Newborn, and Child Health Services in Ethiopia. Multilevel Model Analysis. Int J Prev Med. 2019;10:136

31. Shibanuma A, Yeji F, Okawa S, Mahama E, Kikuchi K, Narh C, et al. The coverage of continuum of care in maternal, newborn and child health: a cross-sectional study of woman-child pairs in Ghana. BMJ Glob Health. 2018 ; 3(4):e000786.

32. Asratie MH, Muche AA, Geremew AB. Completion of maternity continuum of care among women in the post-partum period: Magnitude and associated factors in the northwest, Ethiopia. PLoS One. 2020;15(8): e0237980.

33. Haile D, Kondale M, Andarge E, Tunje A, Fikadu T, Boti N. Level of completion along continuum of care for maternal and newborn health services and factors associated with it among women in Arba Minch Zuria woreda, Gamo zone, Southern Ethiopia: A community based cross-sectional study. PLoS One. 2020;15(6):e0221670.

34. Hamdela B, Getnet $Y$, Abageda M. Predictors of Health Facility Delivery Service Utilization in Lemo District, South Ethiopia: Unmatched Case Control Study. J Preg Child Health. 2015;2:152. https://doi.org/10.4172/2376-127X.1000152.

35. Sakuma S, Yasuoka J, Phongluxa K, Jimba M. Determinants of continuum of care for maternal, newborn, and child health services in rural Khammouane, Lao PDR. PLoS One. 2019:14(4):e0215635.

36. Zambia civil society for poverty reduction. Provinces: Western. http://www csprzambia.org/provinces/western-province/, Accessed December 2020

37. Khatiwada J, Muzembo BA, Wada K, Ikeda S. Dimensions of women's empowerment on access to skilled delivery services in Nepal. BMC Pregnancy Childbirth. 2020;20(1):622.

38. Pulok MH, Sabah MN, Uddin J, Enemark U. Progress in the utilization of antenatal and delivery care services in Bangladesh: where does the equity gap lie? BMC Pregnancy Childbirth. 2016;16(1):200.

39. Okedo-Alex IN, Akamike IC, Ezeanosike OB, Uneke CJ. Determinants of antenatal care utilisation in sub-Saharan Africa: a systematic review. BMJ Open. 2019;9(10):e031890. 
40. Bwalya BB, Mulenga MC, Mulenga JN. Factors associated with postnatal care for newborns in Zambia: analysis of the 2013-14 Zambia demographic and health survey. BMC Pregnancy Childbirth. 2017;17(1):418.

41. Asp G, Pettersson KO, Sandberg J, Kabakyenga J, Agardh A. Associations between mass media exposure and birth preparedness among women in southwestern Uganda: a community-based survey. Glob Health Action. 2014;7(1):22904

42. Gabrysch S, Campbell OMR. Still too far to walk: Literature review of the determinants of delivery service use. BMC Pregnancy Childbirth. 2009;9(1):34.

43. Dankwah E, Zeng W, Feng C, Kirychuk S, Farag M. The social determinants of health facility delivery in Ghana. Reprod Health. 2019;16(1):101.

44. Uganda Bureau of Statistics - UBOS, ICF: Uganda Demographic and Health Survey 2016. In. Kampala, Uganda: UBOS and ICF; 2018.

45. Zambia Statistics Agency, Ministry of Health (MOH) Zambia, and ICF. Zambia Demographic and Health Survey 2018. Lusaka, Zambia, and Rockville, Maryland, USA: Zambia Statistics Agency, Ministry of Health, and ICF; 2019.

\section{Publisher's Note}

Springer Nature remains neutral with regard to jurisdictional claims in published maps and institutional affiliations.

Ready to submit your research? Choose BMC and benefit from:

- fast, convenient online submission

- thorough peer review by experienced researchers in your field

- rapid publication on acceptance

- support for research data, including large and complex data types

- gold Open Access which fosters wider collaboration and increased citations

- maximum visibility for your research: over $100 \mathrm{M}$ website views per year

At $\mathrm{BMC}$, research is always in progress.

Learn more biomedcentral.com/submissions 